\title{
On the shift of cargo on a railway platform under the influence of transverse forces
}

\author{
Khabibulla Turanov ${ }^{1}$, Yadgor Ruzmetov ${ }^{2, *}$ \\ ${ }^{I}$ Tashkent State Technical University named Islam Karimov, University Str, 2, 100174, Tashkent, \\ Uzbekistan \\ ${ }^{2}$ Tashkent Railway Engineering Institute, Temirylchilar Str., 1, 100167, Tashkent, Uzbekistan
}

\begin{abstract}
This paper dwells upon securing solid cargo on a railcar. Transverse cargo shift and forces of elastic securing devices. The authors sought to calculate the shearing force acting on the elastic securing elements and retention bars; the retentive forces that prevent cargo from shifting across the car (the transverse shift); the transverse cargo shift; the force of elastic securing elements as exposed to transverse forces. This study relies on the second law as applicable to relative motion for a frictional constraint, as known in theoretical mechanics. The paper presents the calculated shearing and retentive forces. The authors calculated the equivalent stiffness of elastic securing elements. They thus computed the transverse shift (displacement) of cargo and the elongation of each elastic element that secures the cargo. They also found the forces of elastic securing elements as exposed to transverse forces. It was thus discovered that the forces of the second securing pair were more than double the acceptable limit, which would cause that pair to break during transport. To make the second elastic pair stronger, one might double the number of retention-bar fasteners so that the forces of the second fastening pair wouldn't exceed the limits. An example of calculation is useful in developing a new methodology for calculating the elements of cargo securing on a car. The calculation results proved that to ensure guaranteed safety and reliability of the transportation process, it is necessary to either increase the number of securing elements of the retention bar, or increase the number of elastic securing elements with subsequent recalculation of forces in all elastic securing elements.
\end{abstract}

\section{Introduction}

Papers [1-16] dwell upon securing the cargo on a railway car. For instance, paper [1] investigates the issues of securing the retention bars with fasteners (nails) and notes that the acceptable load on a single $150 \ldots 200(\mathrm{~mm})$ long nail is $\left[R_{\text {nail }}\right]=1.5$. Paper [11] attempts at describing the mathematical model of securing cargo with elastic components as affected by transverse forces; it gives no examples of calculating the parameters of the securing

\footnotetext{
* Corresponding author: yadgor.ruzmetov@ yandex.ru
} 
elements. Papers [12-14] present the mathematical modeling of securing cargo on a gondola as affected by spatial forces. Particularly, these papers provide analytical formulas for calculating the forces acting on the securing elements. Paper [15] presents formulas for calculating the parameters of lashing wires with no regard for the force of pre-twisting. That is despite the fact that every lashing wire that is expected to carry $20(\mathrm{kN})$ of load or more must be pre-twisted when preparing the cargo for shipment by rail. The crucial con of the paper [15] is that the force calculated for each securing point will be the same regardless of its spatial position. Authors [15] do not even use the concept of cargo shift or displacement, although it is such shift during transport that causes nails of retention bars to be pulled out and the lashing wire to sag and/or break. Paper [16] presents research into securing complex-outline cargo on a railway car, where the retention bar is inclined.

Unfortunately, there is little research to date on the transverse shift of cargo that causes flexible securing elements to elongate. That said, calculating the forces of flexible securing elements with adjustment for the number of retention bars and nails is a relevant issue of securing the cargo on a car by the new method [12-14].

Objectives:

- calculate the shearing forces that the elastic securing elements and retention bars are exposed to;

- calculate the retentive forces that prevent the transverse cargo shift;

- calculate the difference between shearing and retentive forces acting across the car;

- find the equivalent stiffness of elastic securing elements adjusted for the physical and geometric parameters of cargo securing;

- calculate the cargo shift across the car as caused by transverse force;

- find the elongation of each elastic securing element;

- find the internal forces of elastic securing elements as exposed to transverse forces.

\section{Method of research}

This research relies on the second law of classical mechanics as applicable to relative motion [5, 12-14].

\section{Mathematical representation of forces}

Use Mathcad [17] to calculate the forces that the elastic securing elements and retention bars are exposed to by the formulas provided in $[5,12,14]$, which can be summarized as follows.

1.1. Calculating the non-Coriolis fictitious forces, the elastic securing elements are exposed to.

Lateral transient Force Inertia $I_{e y}[12,14]$ :

$$
I_{e y}=k_{\text {tol. } y} G
$$

where $k_{\text {tol.y }}=a_{e y} / \mathrm{g}$ is the transverse car dynamics factor (transverse translational acceleration of a loaded car as a fraction of $\mathrm{g}$ ); $a_{e y}$ is the transverse translational acceleration of the car $(0.46 \div 0.55)\left(\mathrm{m} / \mathrm{s}^{2}\right) ; G$ is the gravitational force of the solid cargo $(\mathrm{kN})$.

For the input data: $a_{e y}=0.52 \mathrm{~g}, k_{\text {tol. } y}=a_{e y} / \mathrm{g}=0.52$ and $G=450(\mathrm{kN})$ :

$$
I_{e y}=k_{\text {tol. } y} G=0.52 \cdot 450=234 \text {. }
$$


1.2. Vertical transient Force Inertia $I_{e z}[12,14]$ :

$$
I_{e z}=k_{\text {tol..z }} G,
$$

where $k_{\text {tol. } z}=a_{e z} / \mathrm{g}$ is the vertical car dynamics factor (vertical translational acceleration of a loaded car as a fraction of $\mathrm{g}$ ); $a_{e z}=0.62 \mathrm{~g}$ is the vertical translational acceleration of the car $(0.46 \div 0.66)\left(\mathrm{m} / \mathrm{s}^{2}\right)$.

For the input data: $a_{e z}=0.62 \mathrm{~g}, k_{\mathrm{tol} . z}=a_{e z} / \mathrm{g}=0.62$ and $G=450(\mathrm{kN})$ :

$$
I_{e z}=k_{\mathrm{tol} . \mathrm{z}} G=0.62 \cdot 450=279 \text {. }
$$

1.3. Calculating the aerodynamic drag (wind load) affecting the windward surface area of the solid cargo $[6,12,14,15]$ :

$$
F_{\text {w0 }}=0.5 A_{\text {wind. }}
$$

or

$$
F_{\mathrm{w}}=10^{-3} c \rho_{\mathrm{w}} A_{\text {wind. }} v^{2} / 2 .
$$

Here, 0.5 is a factor of adjustment for the windward surface of the cargo, $\left(\mathrm{kN} / \mathrm{m}^{2}\right) ; A_{\text {wind. }}$. is the windward surface area of the cargo $\left(\mathrm{m}^{2}\right)$ found by the formula:

$$
A_{\text {wind. }}=k_{\mathrm{c}} 10^{-6} \cdot 2 \cdot H \cdot 2 \cdot L,
$$

where $k_{\text {cont. }}$ is the factor of adjustment for the continuity of the windward surface area; $2 \cdot \mathrm{L}$ the length of cargo, $\mathrm{m} ; 2 \cdot \mathrm{H}$ is the height of cargo, $\mathrm{m}$;

$C$ is the factor of adjustment for the windward surface;

$\rho_{\mathrm{w}}$ is the air density $\left(\mathrm{kg} / \mathrm{m}^{3}\right)$;

$v_{\mathrm{w}}$ is the wind speed $(\mathrm{m} / \mathrm{s})$.

For input data $k_{\text {cont. }}=0.65 ; 2 \cdot \mathrm{L}=9.27 \times 10^{3} ; 2 \cdot \mathrm{H}=3.332 \times 10^{2} ; \quad c=0.96$; $\rho_{\mathrm{w}}=1.29 ; v_{\mathrm{w}}=24.8$ obtain:

$A_{\text {wind. }}=k_{\text {cont. }} 10^{-6} \cdot 2 \cdot H \cdot 2 \cdot L=0.65 \cdot 10^{-6} \cdot 3.332 \times 10^{2} \cdot 9.27 \times 10^{3}=20.077\left(\mathrm{~m}^{2}\right)$ :

$F_{\text {w. } 0}=0.5 A_{\text {wind. }}=0.5 \cdot 20.077=10.04$ (equation (11) in [15]) $(\mathrm{kN})$;

$F_{\mathrm{w}}=10^{-3} \cdot 0.96 \cdot 1.29 \cdot 20.1 \cdot(28.4)^{2} / 2=10^{-3} \cdot 20.077 \cdot 806.56 / 2=10.038[6,12](\mathrm{kN})$.

1.4. Friction in the cargo-floor coupling or sliding follows the formula (3.10) in [12]:

$$
\begin{gathered}
F_{\text {fr.coup. } y}=f_{\text {coup }} G\left(1-k_{\mathrm{tol} z}\right) ; \\
F_{\text {fr. } y}=f G\left(1-k_{\mathrm{tol} z}\right) .
\end{gathered}
$$

Here $f_{\text {coup. }}$ is the coupling friction coefficient (usually metal-to-wood friction $f_{\text {coup. }}=$ 0.45 ); $f$ is the sliding friction coefficient, usually $f=0.7 f_{\text {coup. }}$.

For the input data $f=0.7 \cdot 0.45=0.315, k_{\mathrm{tol} . z}=0.62$, and $G=450(\mathrm{kN})$ : 


$$
\begin{gathered}
F_{\text {fr.coup } . y}=0.45 \cdot 450 \cdot(1-0.62)=76.95 \\
F_{\text {fr. } y}=0.315 \cdot 450 \cdot(1-0.62)=53.865
\end{gathered}
$$

1.5. Find the transverse shearing forces $[12,14]$ :

$$
F_{\text {shear. } y}=F_{y}=k_{\text {saf. }}\left(I_{e y}-F_{\mathrm{w}}\right)
$$

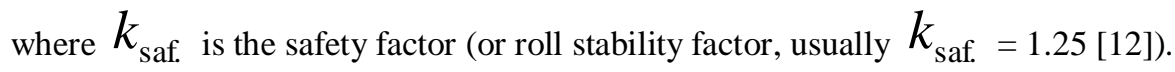

For the input data $k_{\text {saf. }}=1.25, I_{e y}=234$, and $F_{\mathrm{w}}=10(\mathrm{kN})$ :

$$
F_{\text {shear. } y}=F_{y}=1.25(234+10)=305.034 \text {. }
$$

1.6. Find the shearing force that acts on the elastic securing elements across the car; adjust for the coupling and sliding friction, see equation (3.19) in [12]:

$$
\begin{gathered}
\Delta F_{\text {coup. } y}=F_{\text {shear. } y}-F_{\text {fr.coup } . y}, \\
\Delta F_{\text {fr. } y}=F_{\text {shear. } y}-F_{\text {fr. } y} .
\end{gathered}
$$

Substitute the input data $F_{\text {shear. } y}=305.034, F_{\text {fr.coup. } y}=76.95$, and $F_{\text {fr. } y}=53.865$ $(\mathrm{kN})$ in the latest equations to get $(\mathrm{kN})$ :

$$
\begin{gathered}
\Delta F_{\text {coup. } y}=F_{\text {shear. } y}-F_{\text {fr.coup. } y}=305.034-76.95=228.084 \\
\Delta F_{\text {fr. } y}=F_{\text {shear. } y}-F_{\text {fr. } y}=305.034-53.865=251.169 .
\end{gathered}
$$

1.7. Find the retention-bar response adjusted for the car floor condition and weather; to that end, use the formula (4.5) in [2] and Table 32 [15]:

$$
R_{\text {bar. } y}=k_{1} n_{\text {nail.y }} n_{\text {bar. } y}\left[R_{\text {nail }}\right]
$$

where $k_{1}=0.6$ is the holding strength of retention bars adjusted for the car floor condition, pcs, usually assumed to equal $0.5 \div 0.8$ provided that the cargo outline effectively prevents using more nails; else, $1.5 \div 2.0$;

$n_{\text {nail.y }}=14$ is the assumed required number of fasteners (nails) for each retention bar (pcs);

$n_{\text {bar. } y}=4$ is the assumed number of retention bars as shown in the cargo placement and securing diagram, pcs;

$\left[R_{\text {nail }}\right]=1.08$ is the acceptable limit of force acting on a single $\varnothing 6 \mathrm{~mm}, 150$ to 200 mm long fastener (nails), $\mathrm{kN}$ (Table 32 [15]); according to [2] $\left[R_{\text {nail }}\right]=1.5$.

Substitute this data in the equation (10) to find the retention-bar response for the assumed number of nails $(\mathrm{kN})$ :

$$
R_{\text {bar. } y}=k_{1} n_{\text {nail. } y} n_{\text {bar. } y}\left[R_{\text {nail }}\right]=0.6 \cdot 14 \cdot 4 \cdot 1.08=36.288
$$




\section{Research results}

\section{Calculation of forces in fasteners taking into account the shift of the load}

Use Mathcad [17] to calculate the forces that the elastic securing elements and retention bars are exposed to by the formulas provided in [12, 14], which can be summarized as follows.

2.1. Let us project the force in elastic securing elements onto the transverse axis of the car and adjust it for the pre-twisting force of the lashing wire at $d=6(\mathrm{~mm}) n=8(\mathrm{pcs}), R 0$ $=20(\mathrm{kN})$ in either direction as the retentive forces that prevent the cargo from shifting across the car; to that end, use the following formulas (see equation (19) in [14]) [12]:

$$
F R 0_{y 1}=R 0 \times\left(\frac{b_{i}}{l_{i}}+\frac{b_{i p}}{l_{i p}}\right) ; F R 0_{y 2}=R 0 \times\left(\frac{b_{i a}}{l_{i a}}+\frac{b_{i a p}}{l_{i a p}}\right), \quad(i=\overline{1,6}),
$$

where $b_{i}$ and $b_{i p}, l_{i}$ and $l_{i p}, b_{i a}$ and $b_{i a p}, l_{i a}$ and $l_{i a p}$ are drawing-based calculated projections of elastic securing elements in either direction onto the transverse axis of the car; and the securing device length (m). Table. 1 summarizes these geometric parameters of the securing devices.

Table 1. Geometry of the securing devices (m)

\begin{tabular}{|c|c|c|c|c|c|c|c|}
\hline $\begin{array}{l}\text { Designation } \\
\text { of the } \\
\text { securing }\end{array}$ & $\begin{array}{l}\text { Direction } \\
\text { of }\end{array}$ & \multicolumn{6}{|c|}{$\begin{array}{l}\text { The values of the projections of the securing devices and } \\
\text { their length }(\mathrm{m})\end{array}$} \\
\hline$b_{i}$ & & 0.608 & 1.043 & 0.174 & 0.174 & 0.174 & 0.174 \\
\hline$l_{i}$ & & 1.467 & 1.087 & 1.06 & 1.432 & 0.952 & $\begin{array}{c}12.07 \\
8\end{array}$ \\
\hline$b_{i p}$ & & 0.869 & 1.912 & 0.174 & 0.174 & 0.174 & 0 \\
\hline$l_{i p}$ & & 1.786 & 2.515 & 1.662 & 1.06 & 1.292 & 0 \\
\hline$b_{i p}$ & & 1.095 & 1.043 & 0.174 & 0.174 & 0.174 & 0.174 \\
\hline$l_{i p}$ & The other & 1.497 & 1.087 & 1.06 & 1.432 & 0.952 & 2.078 \\
\hline$b_{i a p}$ & & 0.869 & 1.912 & 0.174 & 0.174 & 0.174 & 0 \\
\hline$l_{\text {iap }}$ & & 1.851 & 2.515 & 1.662 & 1.06 & 1.292 & 0 \\
\hline
\end{tabular}

Substitute the data of Table. 1 in (11), which are the source data adjusted for the cargo placement asymmetry with respect to the transverse axis of the car, obtain $(\mathrm{kN})$ :

$$
\text { FROy } 1=20 \cdot\left(\begin{array}{l}
\frac{0.608}{1.467}+\frac{0.869}{1.786}+\frac{1.043}{1.087}+\frac{1.912}{2.515}+\frac{0.174}{1.06}+\frac{0.174}{1.662}+\frac{0.174}{1.432}+ \\
+\frac{0.704}{1.06}+\frac{0.174}{0.952}+\frac{0.174}{1.292}+\frac{0.174}{2.078}
\end{array}\right)=71.514
$$


or $F R 0$ ret. $y 1=F R 0_{y 1}=71.514$;

FROy $2=20 \cdot\left(\begin{array}{l}\frac{1.005}{1.497}+\frac{0.869}{1.851}+\frac{1.043}{1.087}+\frac{1.912}{2.515}+\frac{0.174}{1.6}+\frac{0.174}{1.662}+\frac{0.174}{1.432}+ \\ +\frac{0.174}{1.06}+\frac{0.174}{0.952}+\frac{0.174}{1.292}+\frac{0.174}{2.078}\end{array}\right)=71.171$ or $F R 0$ ret. $y 2=R 0_{y 2}=71.171$.

Apparently, $F R 0_{\text {ret. } y 1}=F R 0_{y 1}$ and $F R 0_{\text {ret. } y 2}=R 0_{y 2}$ are the elastic retentive forces of the securing elements in either direction, arising from pre-twisting.

2.2. Find the difference in the projections of wire pre-twisting in either direction by the formula:

$$
F R 0_{y 0}=F R 0_{\text {ret. } y 1}-F R 0_{\text {ret. } y 2 .}
$$

Substitute the input data as found by (11) in (12) to obtain $(\mathrm{kN})$ :

$$
F R 0_{y 0}=F R 0_{\text {ret. } y 1}-F R 0_{\text {ret. } y 2}=71.514-71.171=0.343 .
$$

Thus, the total projection of elastic forces onto the transverse axis is positive for the case under consideration. This means the elastic securing elements are asymmetric in either direction.

2.3. Project the force (elastic forces) of wire pre-twisting $R 0$ in either direction onto the vertical axis by the formulas $[12,14]$ :

$$
F R 0_{z 1 y}=R 0 \cdot\left(\frac{h_{i}}{l_{i}}+\frac{h_{i p}}{l_{i p}}\right) ; F R 0_{z 2 y}=R 0 \cdot\left(\frac{h_{i a}}{l_{i a}}+\frac{h_{i a p}}{l_{i a p}}\right) \quad(i=\overline{1,6}),
$$

where $h_{i}$ and $h_{i p}, l_{i}$ and $l_{i p}, h_{i a}$ and $h_{i a p}, l_{i a}$ and $l_{i a p}$ are drawing-based calculated projections of elastic securing elements in either direction onto the vertical axis of the car; and the length of these elements (m). Table. 2 summarizes these geometric parameters of

\begin{tabular}{|c|c|c|c|c|c|c|c|}
\hline \multirow{2}{*}{$\begin{array}{c}\begin{array}{c}\text { Designation of } \\
\text { the } \\
\text { securing devices }\end{array} \\
h_{i}\end{array}$} & Direction of & \multicolumn{6}{|c|}{ The values of the projections of the securing devices and their length $(\mathrm{m})$} \\
\hline & \multirow{4}{*}{$\begin{array}{c}\text { One } \\
\text { direction }\end{array}$} & 0.605 & 0.302 & 0.907 & 0.907 & 0.907 & 0.907 \\
\hline$l_{i}$ & & 1.467 & 1.087 & 1.06 & 1.432 & 0.952 & 12.078 \\
\hline$h_{i p}$ & & 1.008 & 1.008 & 0.907 & 0.907 & 0.907 & 0 \\
\hline$l_{i p}$ & & 1.786 & 2.515 & 1.662 & 1.06 & 1.292 & 0 \\
\hline$h_{i a}$ & \multirow{4}{*}{$\begin{array}{l}\text { The other } \\
\text { direction }\end{array}$} & 0.605 & 0.302 & 0.907 & 0.907 & 0.907 & 0.907 \\
\hline$l_{i a}$ & & 1.497 & 1.087 & 1.06 & 1.432 & 0.952 & 2.078 \\
\hline$h_{\text {iap }}$ & & 1.008 & 1.008 & 0.907 & 0.907 & 0.907 & 0 \\
\hline$l_{i a p}$ & & 1.851 & 2.515 & 1.662 & 1.06 & 1.292 & 0 \\
\hline
\end{tabular}
the securing devices.

Table 2. Geometry of the securing devices (m)

1n the formula (13), which are the input data, and keep in mind that $R 0=20(\mathrm{kN})$ to 
obtain $(\mathrm{kN})$ :

$$
\begin{gathered}
F R O z 1 y=20 \cdot\left(\begin{array}{l}
\left.\frac{0.605}{1.467}+\frac{1.008}{1.786}+\frac{0.302}{1.087}+\frac{1.008}{2.515}+\frac{0.907}{1.06}+\frac{0.907}{1.662}+\frac{0.907}{1.432}+\frac{0.907}{1.06} \ldots\right) \\
+\frac{0.907}{0.952}+\frac{0.907}{1.252}+\frac{0.907}{2.078}+0
\end{array}\right)=132.765 \\
F R O z 2 y=20 \cdot\left(\begin{array}{l}
\left.\frac{0.605}{1.467}+\frac{1.008}{1.851}+\frac{0.302}{1.087}+\frac{1.008}{2.515}+\frac{0.907}{1.06}+\frac{0.907}{1.662}+\frac{0.907}{1.432}+\frac{0.907}{1.06} \ldots\right) \\
+2 \frac{0.907}{0.952}+\frac{0.907}{1.292}+\frac{0.907}{2.078}+0
\end{array}\right)=132.367 .
\end{gathered}
$$

Apparently, the elastic forces arising from the pre-twisting of lashing wire in either direction are of the following values as projected onto the vertical axis $(\mathrm{kN}): R 0 z 1 y=$ $F R 0 z 1 y=132.765$ и $R 0 z 02 y=F R 0 z 2 y=132.367$.

2.4. Find the total of transverse retentive forces acting on the cargo securing devices and adjusted for the sliding friction $F_{\text {fr.y }}$ as a function of the cargo gravity, force of wire pre-

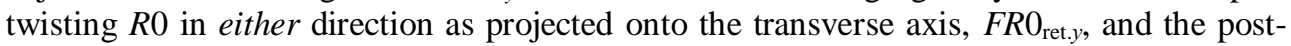
shifting sliding friction as a function of the pre-twisting force $R 0$ projected onto the vertical axis in one direction $F R 0_{\text {fr. } y}$; to that end, use the formula below (see $(3.18$, a) in [12]) [14]:

$$
\Delta F R 0_{\text {ret } y k}=F R 0_{\text {ret } . y 0 k}+R_{\text {bar. } y, \quad(k=1,2)}
$$

where $\triangle F R 0_{\text {ret.yok }}$ are the pre-twisting force $R 0$ as projected onto the transverse axis in one direction only:

$$
F R 0_{\text {ret. } y 0 k}=F_{\text {fr. } y}+F R 0_{\text {ret. } y k}+\Delta F R 0_{\text {fr. } y k}
$$

given that in there, $\Delta F R 0_{\text {fr.yk }}$ is the sliding friction as a function of the post-shifting pretwisting force $R 0$ as projected onto the vertical axis:

$$
F R 0_{\text {fr. } . y k}=f \times F R 0_{z k y}(\mathrm{k}=1.2) .
$$

Substitute the input data found by the equations (10), (11), and (13) in the latest formulas to find the total of transverse retentive forces in one direction $(\mathrm{kN})$ :

$$
\begin{gathered}
F R 0_{\text {fr. } y 1}=f \times F R 0_{z 1 y}=0.315 \cdot 132.765=41.821 ; \\
\Delta F R 0_{\text {ret. } y 01}=F_{\text {fr. } y}+F R 0_{\text {ret. } y 1}+\Delta F R 0_{\text {fr. } y 1}=53.865+71.514+41.821=167.2 ; \\
\Delta F R 0_{\text {ret. } y 1}=F R 0_{\text {ret. } y 01}+R_{\text {bar. } y}=167.2+36.288=203.488 .
\end{gathered}
$$

Similarly, the total of transverse retentive forces in the other direction is $(\mathrm{kN})$ :

$$
\begin{aligned}
F R 0_{\text {fr. } y 2}=f \times F R 0_{z 2 y} & =0.316 \cdot 132.367=41.696 ; \\
F R 0_{\text {ret } y 02}=F F_{\text {fr. } y}+F R 0_{\text {ret. } y 2}+\Delta F R 0_{\text {fr. } y 2} & =53.865+71.171+41.696=166.731 ; \\
\Delta F R 0_{\text {ret. } y 2}=F R 0_{\text {ret } . y 02}+R_{\text {bar. } y} & =163.583+36.288=203.019 .
\end{aligned}
$$

Apparently, the difference between the shearing force $F_{\text {shear.y }}$ and the retentive forces $\triangle F R 0_{\text {coup.ret. } y 1}$ and $R 0_{\text {bar. } y}$ is positive. This is confirmed by the transversal cargo shift that causes the nails of retention bars to be pulled out.

2.5. Find the difference $\triangle F R 0_{y}$ between the shearing transverse forces $F_{\text {shear.y }}$ and the retentive transverse forces $\triangle F R 0_{\text {ret.y }}$ acting on the securing devices; to that end, use the formula below (see (3.19) in [12] and (14) in [14]):

$$
\Delta F R 0_{y}=F_{\text {shear. } y}-\Delta F R 0_{\text {ret. } y .}
$$

Substitute the input data as found by the equations (7) and (14) in (17) to find the transverse forces acting on the securing elements in either direction $(\mathrm{kN})$ :

$$
\Delta F R 0_{y 1}=F_{\text {shear. } y}-\Delta F R 0_{\text {ret. } y 1}=305.034-203.488=101.546 ;
$$




$$
\Delta F R 0_{y 2}=F_{\text {shear } y}-\Delta F R 0_{\text {ret. } y 2}=305.034-203.019=102.014 .
$$

Apparently, the difference between the shearing force $F_{\text {shear.y }}$ and the retentive force $\triangle F R 0_{\text {ret. } y}$ is positive. This means that cargo will definitely be displaced across the car, pulling out the nails of retention bars and causing these bars to turn over at the farther edge (in relation to the cargo face). Whether the retention bars are nailed to the sides of the cargo does not matter. In that case, the retention bars will turn over at one of the edges.

2.6. Find the possible cargo shift across the car as induced by the coplanar force system $\Delta F R 0 y$ [12]. Consider the empirical evidence that shows elastic securing elements sag as the load (cargo) shifts by less than half the width of the retention bar; if no bar is used, sagging happens where the shearing forces exceed the retentive ones. For further calculation, assume the initial cargo-and-bar shift as $\Delta 1 y 0=0.006(\mathrm{~m})$, bearing in mind that a retention bar is usually $0.005 \ldots 0.150(\mathrm{~m})$ wide.

Also, note that the elastic securing elements in the other direction are disengaged, i.e. they are sagging. Thus, the elastic securing elements and retention bars of only one direction are engaged in retaining the cargo to keep it from moving across the car.

2.7. Find the transverse equivalent stiffness of the securing elements $c_{\text {equv. } y}$ adjusted for the physical and geometric parameters of cargo securing $\left(E, n_{i}, d_{i} a_{i}, b_{i}, h_{i}\right.$, and $\left.l_{i}\right)$ at the sliding friction coefficient $f$ (i.e. after the cargo has shifted). To that end, use the equations below (see (3.92) in [12]) [14]:

$$
\begin{gathered}
c_{\text {equv. } y k}=7.854 d_{i}^{2} \cdot C 0_{y k} \quad(\text { where } k=1,2) . \\
\text { where } C 0_{y 1}=\sum_{i=1}^{n_{\mathrm{p}}} \frac{n_{i}}{l_{i}}\left(f \frac{h_{i}}{l_{i}}+\frac{b_{i}}{l_{i}}\right) \frac{b_{i}}{l_{i}}+\sum_{i=1}^{n_{\mathrm{p}}} \frac{n_{i p}}{l_{i p}}\left(f \frac{h_{i p}}{l_{i p}}+\frac{b_{i p}}{l_{i p}}\right) \frac{b_{i p}}{l_{i p}} \\
C 0_{y 2}=\sum_{i=1}^{n_{\mathrm{p}}} \frac{n_{i a}}{l_{i a}}\left(f \frac{h_{i a}}{l_{i a}}+\frac{b_{i a}}{l_{i a}}\right) \frac{b_{i a}}{l_{i a}}+\sum_{i=1}^{n_{\mathrm{p}}} \frac{n_{i a p}}{l_{i a p}}\left(f \frac{h_{i a p}}{l_{i a p}}+\frac{b_{i a p}}{l_{i a p}}\right) \frac{b_{i a p}}{l_{i a p}} .
\end{gathered}
$$

Calculate the equivalent stiffness of the transverse securing elements $c_{\text {equv.y }}$ which for the reason of transversal axial asymmetry in either direction at $f=0.315, d_{i}=6(\mathrm{~mm}), n_{i}=8$ (pcs); applying the formula (18) returns $(\mathrm{kN} / \mathrm{m})$ :

$$
\begin{aligned}
& C 0 y 1=\left[\frac{8}{1.467} \cdot\left(0.315 \cdot \frac{0.605}{1.467}+\frac{0.608}{1.467}\right)\right] \cdot \frac{0.608}{1.467}+\left[\frac{8}{1.786} \cdot\left(0.315 \cdot \frac{1.008}{1.786}+\frac{0.869}{1.786}\right)\right] \cdot \frac{0.869}{1.786} \ldots \\
& +\left[\frac{8}{1.087} \cdot\left(0.315 \cdot \frac{0.605}{1.087}+\frac{1.043}{1.087}\right)\right] \cdot \frac{1.043}{1.087}+\left[\frac{8}{2.515} \cdot\left(0.315 \cdot \frac{1.008}{2.515}+\frac{1.912}{2.515}\right)\right] \cdot \frac{1.912}{2.515} \ldots \\
& +\left[\frac{8}{1.06} \cdot\left(0.315 \cdot \frac{0.302}{1.06}+\frac{0.74}{1.06}\right)\right] \cdot \frac{0.174}{1.06}+\left[\frac{8}{1.662} \cdot\left(0.315 \cdot \frac{0.907}{1.662}+\frac{0.174}{1.662}\right)\right] \cdot \frac{0.174}{1.662} \ldots \\
& +\left[\frac{8}{1.432} \cdot\left(0.315 \cdot \frac{0.907}{1.432}+\frac{0.174}{1.432}\right)\right] \cdot \frac{0.174}{1.432}+\left[\frac{8}{1.06} \cdot\left(0.315 \cdot \frac{0.907}{1.06}+\frac{0.174}{1.06}\right)\right] \cdot \frac{0.174}{1.06} \ldots \\
& +\left[\frac{8}{0.952} \cdot\left(0.315 \cdot \frac{0.907}{0.952}+\frac{0.174}{0.952}\right)\right] \cdot \frac{0.174}{0.952}+\left[\frac{8}{1.292} \cdot\left(0.315 \cdot \frac{0.907}{1.292}+\frac{0.174}{1.292}\right)\right] \cdot \frac{0.174}{1.292} \ldots \\
& +\left[\frac{8}{2.078} \cdot\left(0.315 \cdot \frac{0.907}{2.078}+\frac{0}{2.078}\right)\right] \cdot \frac{0}{2.078}=14.762
\end{aligned}
$$

Substitute C0y1 $=14.762$ in $(19)$ to obtain $(\mathrm{kN} / \mathrm{m}): c_{\text {equv. } y 1}=7.854 \cdot \mathrm{d}^{2} \cdot \mathrm{C} 0 \mathrm{y} 1=4.174 \times 10^{3}$; 


$$
\begin{aligned}
& \text { C0y2 }=\left[\frac{8}{1.467} \cdot\left(0.315 \cdot \frac{0.605}{1.467}+\frac{1.095}{1.467}\right)\right] \cdot \frac{1.095}{1.467}+\left[\frac{8}{1.851} \cdot\left(0.315 \cdot \frac{1.008}{1.851}+\frac{0.869}{1.851}\right)\right] \cdot \frac{0.869}{1.851} \ldots \\
& +\left[\frac{8}{1.087} \cdot\left(0.315 \cdot \frac{0.302}{1.087}+\frac{1.043}{1.087}\right)\right] \cdot \frac{1.043}{1.087}+\left[\frac{8}{2.515} \cdot\left(0.315 \cdot \frac{1.008}{2.515}+\frac{1.912}{2.515}\right)\right] \cdot \frac{1.912}{2.515} \ldots \\
& +\left[\frac{8}{1.06} \cdot\left(0.315 \cdot \frac{0.907}{1.06}+\frac{0.174}{1.06}\right)\right] \cdot \frac{0.174}{1.06}+\left[\frac{8}{1.662} \cdot\left(0.315 \cdot \frac{0.907}{1.662}+\frac{0.174}{1.662}\right)\right] \cdot \frac{0.174}{1.662} \ldots \\
& +\left[\frac{8}{1.432} \cdot\left(0.315 \cdot \frac{0.907}{1.432}+\frac{0.174}{1.432}\right)\right] \cdot \frac{0.174}{1.432}+\left[\frac{8}{1.292} \cdot\left(0.315 \cdot \frac{0.907}{1.292}+\frac{0.174}{1.292}\right)\right] \cdot \frac{0.174}{1.292} \ldots \\
& +\left[\frac{8}{0.952} \cdot\left(0.315 \cdot \frac{0.907}{0.952}+\frac{0.174}{0.952}\right)\right] \cdot \frac{0.174}{0.952}+\left[\frac{8}{1.292} \cdot\left(0.315 \cdot \frac{0.907}{1.292}+\frac{0.174}{1.292}\right)\right] \cdot \frac{0.174}{1.292} \ldots \\
& +\left[\frac{8}{2.078} \cdot\left(0.315 \cdot \frac{0.907}{2.078}+\frac{0.907}{2.078}\right)\right] \cdot \frac{0.907}{2.078}=14.614
\end{aligned}
$$

Substitute $\mathrm{C} 0 y 2=14.614$ in $(18)$ to obtain $(\mathrm{kN} / \mathrm{m}): c_{\text {equv. } y 2}=7,854 \cdot \mathrm{d}^{2} \cdot \mathrm{C} 0 \mathrm{y} 2=4.132$ $\times 10^{3}$.

2.8. To find the transverse cargo shift induced by the transverse force system after the retention bars have been pulled out in one direction use the formula $[12,14]$ :

$$
\Delta y_{k}=\Delta F R 0_{y k} / c_{\text {equv. } y k}+\Delta y_{0} \quad(\text { where } k=1.2) .
$$

Substitute the input data as $\Delta F R 0 y 1=98.398$ and $\Delta F R 0 y 2=105.162(\mathrm{kN}) ; \Delta y 0=$ 0.006, the assumed initial cargo-and-bar displacement (m), in (19) to find post-pullout cargo shift for either direction $(\mathrm{m})$ : $\mathrm{mm})$;

$\Delta y 1=\Delta F R 0 y 1 / \mathrm{c}_{\text {equv. } y 1}+\Delta y 0=101.546 / 4174+0.006=0.024+0.006=0.03$ (or 30 $\mathrm{mm})$.

$\Delta y 2=\Delta F R 0 y 2 / \mathrm{c}_{\text {equv. } y 2}+\Delta y 0=102.014 / 4132+0.006=0.025+0.006=0.031$ (or 31

2.9. Find the lashing wire load tolerance by the formula [12]:

$$
R_{\mathrm{tol} i}=\left[R_{i 0}\right]+R 0_{i} \quad(i=1 \ldots 6)
$$

where $\left[R_{i 0}\right]$ is the permissible tensile stress as a function of the number of threads $n_{i}(8$ pcs) and diameter $d_{i}(6 \mathrm{~mm})$, usual $24.8(\mathrm{kN})$, see Table 30, p. 79 in [15]);

$R 0_{i}$ is the pre-twisting tension, normally $20(\mathrm{kN})[12,14]$.

Assume the following for the value of maximum permissible load for each wire $(\mathrm{kN})$ :

$$
R_{\mathrm{tol} i}=\left[R_{i 0}\right]+R 0_{i}=24.8+24=44.8 .
$$

2.10. Find the elongation of each wire in either direction as a function of the transverse cargo shift (see (22) in [14]):

$$
\Delta l_{i y}=\Delta y_{0 i} \times \frac{b_{i}}{l_{i}} ; \quad \Delta l_{i p y}=\Delta y_{0 p i} \times \frac{b_{i p}}{l_{i p}} .
$$

Table 3 presents the calculated elongations of securing elements. 
Table 3. Calculated elongation of the securing elements in either direction (m)

\begin{tabular}{|c|c|c|c|c|c|c|c|}
\hline $\begin{array}{l}\text { Designation } \\
\text { of } \\
\text { elongation }\end{array}$ & $\begin{array}{l}\text { Direction } \\
\text { of }\end{array}$ & \multicolumn{6}{|c|}{ Elongations in the securing elements (m) } \\
\hline \multirow{2}{*}{$\frac{\Delta l_{i y}}{\Delta l_{i y p}}$} & \multirow{2}{*}{$\begin{array}{c}\text { One } \\
\text { direction }\end{array}$} & $\frac{\Delta l_{1 y}}{\Delta l_{1 y p}}$ & $\frac{\Delta l_{2 y}}{\Delta l_{2 y p}}$ & $\frac{\Delta l_{3 y}}{\Delta l_{3 y p}}$ & $\frac{\Delta l_{4 y}}{\Delta l_{4 y p}}$ & $\frac{\Delta l_{5 y}}{\Delta l_{5 y p}}$ & $\frac{\Delta l_{6 y}}{\Delta l_{6 y p}}$ \\
\hline & & $\frac{0.013}{0.015}$ & 0.029 & 0.00497 & 0.00368 & 0.00553 & 0.00254 \\
\hline \multirow{2}{*}{$\frac{\Delta l_{\text {iay }}}{\Delta l_{\text {iapy }}}$} & \multirow{2}{*}{$\begin{array}{l}\text { The other } \\
\text { direction }\end{array}$} & $\frac{\Delta l_{1 a y}}{\Delta l_{1 a p y}}$ & $\frac{\Delta l_{2 a y}}{\Delta l_{2 a p y}}$ & $\frac{\Delta l_{3 a y}}{\Delta l_{3 a p y}}$ & $\frac{\Delta l_{4 a y}}{\Delta l_{4 a p y}}$ & $\frac{\Delta l_{5 a y}}{\Delta l_{5 a p y}}$ & $\frac{\Delta l_{6 a y}}{\Delta l_{6 a p y}}$ \\
\hline & & $\frac{0.013}{0.014}$ & $\frac{0.029}{0.023}$ & $\frac{0.005}{0.0032}$ & $\frac{0.00372}{0.005}$ & $\frac{0.0056}{0.00413}$ & $\frac{0.00257}{0}$ \\
\hline
\end{tabular}

2.11. Find the forces of the elastic securing elements as affected by transverse forces by the following formula (see (3.93) in [12] and (22) in [14]):

$$
\begin{gathered}
R_{i y}=R_{\text {elast } . i}=7.854 \times d_{i}^{2} \times \frac{n_{i}}{l_{i}} \times \Delta l_{i y} \leq\left[R_{i 0}\right](i=1 \ldots 6) ; \\
R_{i p y}=R_{\text {elast. } i p}=7.854 \times d_{i p}^{2} \times \frac{n_{i p}}{l_{i p}} \times \Delta l_{i p y} \leq\left[R_{i p 0}\right]
\end{gathered}
$$

where $7.854=\pi \mathrm{E} 10^{-6} / 4$ is a factor of adjustment for elastic modulus of the securing elements as affected by wire twisting (normally $E=1 \cdot 10^{7}$ for annealed wire) [12, p. 200], $\left(\mathrm{kN} / \mathrm{m}^{2}\right)$;

$\left[R_{i 0}\right]$ - is the permissible value of force in the $i$ th securing element, which can be found in Table 30 [15] as a function of the number of threads $n_{i}(8 \mathrm{pcs})$ and diameter $d_{i}(6$ $\mathrm{mm})$ with adjustment for the pre-twisting forces $R 0_{i}=20(\mathrm{kN})$, i.e. $\left[R_{i 0}\right]=\left[R_{i}\right]+R 0_{i}=$ $24.8+20=44.8(\mathrm{kN})$.

Table 4 presents the calculated forces of securing elements.

Table 4. Calculated forces of the securing elements in either direction $(\mathrm{kN})$

\begin{tabular}{|l|l|l|l|l|l|l|l|}
\hline $\begin{array}{c}\text { Designations of } \\
\text { forces in the } \\
\text { securing } \\
\text { elements }\end{array}$ & $\begin{array}{l}\text { Direction of } \\
\text { securing }\end{array}$ & \multicolumn{6}{|c|}{ Forces in the securing elements $(\mathrm{kN})$} \\
\hline \multirow{2}{*}{$\frac{R_{\text {iy }}}{R_{\text {iyp }}}$} & $\begin{array}{l}\text { One } \\
\text { direction }\end{array}$ & $\frac{R_{1 y}}{R_{1 y p}}$ & $\frac{R_{2 y}}{R_{2 y p}}$ & $\frac{R_{3 y}}{R_{3 y p}}$ & $\frac{R_{4 y}}{R_{4 y p}}$ & $\frac{R_{5 y}}{R_{5 y p}}$ & $\frac{R_{6 y}}{R_{6 y p}}$ \\
\cline { 3 - 9 } & $\frac{19.34}{18.7}$ & $\frac{60.6}{20.74}$ & $\frac{10.61}{4.32}$ & $\frac{5.81}{10.61}$ & $\frac{13.14}{7.15}$ & $\frac{2.76}{0}$ \\
\hline$\frac{R_{\text {iay }}}{R_{\text {iapy }}}$ & The other & $\frac{R_{\text {lay }}}{R_{1 \text { apy }}}$ & $\frac{R_{2 a y}}{R_{2 a p y}}$ & $\frac{R_{3 a y}}{R_{3 a p y}}$ & $\frac{R_{4 a y}}{R_{4 a p y}}$ & $\frac{R_{5 a y}}{R_{5 a p y}}$ & $\frac{R_{6 a y}}{R_{6 a p y}}$ \\
\cline { 3 - 9 } & $\frac{19.61}{17.61}$ & $\frac{61.32}{20.98}$ & $\frac{10.74}{4.37}$ & $\frac{5.88}{10.74}$ & $\frac{13.3}{7.23}$ & $\frac{2.8}{0}$ \\
\hline
\end{tabular}


Analysis of these estimates showed that the forces of all flexible securing components (except the second pair) in either direction was significantly below the acceptable limits in either direction when exposed to transverse forces $(24.8+20=44.8(\mathrm{kN}))$. Thus, these securing components were redundantly strong. However, the forces of the second securing pair were more than double the acceptable limit $\left(R_{2 y} \approx R_{2 a y} \approx 61>44.8(\mathrm{kN})\right)$. This pair was not sufficiently strong and would break during transport. To make the second elastic pair sufficiently strong, forces acting in the elastic securing elements must be recalculated. Calculations show that merely doubling the number of nails $n_{\text {nail. }}$ per retention bar must suffice $\left(n_{\text {nail. }}=28\right.$ instead of $\left.n_{\text {nail. }}=14\right)$. Else, the safety and reliability of transport will be compromised.

Clearly, unlike in [15], the proposed method for estimating the elastic securing elements (i) makes adjustments for the pre-twisting forces of the wire, (ii) can be used to calculate the transverse cargo shift $\Delta y$, see (19), (iii) can be used to find the elongation of each elastic securing element $\Delta l$ as a function of its geometry, see (20), and then find the forces of each $i$ th lashing element $R_{i}$ (where $i$ is the number of securing elements in either direction that are exposed to transverse forces, pcs), see (21) and (22).

\section{Conclusion}

1. The authors hereof calculated the shearing force acting on the elastic elements and retention bars securing the cargo; they also estimated the retentive forces that prevent transverse cargo shift with adjustments for wire pre-twisting forces.

2. They further calculated the transverse cargo shift induced by transverse forces as a function of the equivalent stiffness of elastic securing elements with adjustments for the physical and geometric parameters of the securing devices.

3. Then they found the elongation of each elastic securing element and estimated the forces arising in such elements when exposed to transverse forces. It was thus discovered that the forces of the second securing pair were more than double the acceptable limit $\left(R_{2 y} \approx R_{2 a y} \approx 61>44.8(\mathrm{kN})\right)$, which would cause that pair to break during transport. To make the second elastic pair sufficiently strong, forces acting in the elastic securing elements must be recalculated. Calculations show that merely doubling the number of nails $n_{\text {nail. }}$ per retention bar must suffice $\left(n_{\text {nail. }}=28\right.$ instead of $\left.n_{\text {nail. }}=14\right)$, so that the forces of the second pair would not exceed the limits $\left(R_{2 y} \approx R_{2 a y} \approx 43<44.8\right.$ $(\mathrm{kN})$ ).

4. The calculation example is useful when developing a new methodology for calculating the cargo securing elements on a car.

5. The calculation results proved that to ensure guaranteed safety and reliability of the transportation process, it is necessary to either increase the number of fasteners of the retention bar or increase the number of elastic fasteners, followed by the recalculation of forces in all elastic securing elements.

\section{References}

1. N. Anderson, P. Anderson, R. Bylander, S. Sokjer-Petersen, and B. Zether, VINNOVA Report. Rapport VR 2004:05 (2004). Available at: http://www.vinnova.se/upload / EPiStorePDF /vr-04-05.pdf.

2. M. Johanson, P. Andersson, Equipment for Efficient Cargo Securing and Ferry Fastening of Vehicles/NVF-rapporter/Vä gverket (2004). Available at: http://www.nvfnorden.org/lisalib/getfile.aspx?itemid=1593

3. Driver's Handbook Cargo Securement. A Guide to the North American Cargo 
Securement Standard. Available at: http://www.highwaystarmagazine.com/images /Driver_Handbook.pdf

4. European Best Practice Guidelines on Cargo Securing for Road Transport. Available at: http://ec.europa.eu/transport/roadsafety/vehicles/best_practice_guidelines_en.htm

5. K.L. Komarov, A.F. Yashin, Teoreticheskaia makhanika $v$ zadachakh gruzovykh perevozok (Novosibirsk, Nauka Publ., 2004) (in Russ.)

6. J.D. Priddy, R.A. Jones, SAE Technical 2005-01-3516 (2005)

7. J.R. Billing, Industry Practices for Loading and Securing Cargo in Vans and other Closed Trucks (Spring: Ontario Trucking Association, 2006)

8. E. Hildebrand, M.Balsom, and F.R. Wilson, Transportation Research Board Annual Meeting 06-1232 (2006).

9. Load Securement for Palletized Freight in Closed Trucks - From an Enforcement Perspective. dPE, Inc. «The Science of Preventing Freight Damage» (2007)

10. Vertical cylinder handling and transportation AIGA 038/06. Available at: http://www.asiaiga.org.

11. E.N. Timukhina, Transport Problems 3(3), $65-68$ (2008).

12. Kh.T. Turanov, Teoreticheskaia makhanika $v$ zadachakh gruzovykh perevozok. (Novosibirsk, Nauka Publ., 2009) (in Russ.).

13. Kh. Turanov, Global Journal of Research in Engineering (GJRE-A), X(13), 7-16 (2013).

14. Kh.Turanov, Science and Technology 1(5), 5-14 (2015). DOI: 10.5923/j.scit.2015 0501.02) (ID: 104000126).

15. SMGS «Technicheskie usloviia razmeshcheniia i krepleniia gruzov». Prilozdeniie 3. Available at:http://osjd.org/doco/public/ru

16. Kh. Turanov, Y. Ruzmetov, N. Vlasova, E3S Web of Conferences. 135, 02006 (2019). https://doi.org/10.1051/e3sconf/201913502006

17. Ilinykh, A. Manakov, A. Abramov, S. Kolarzh, MATEC Web of Conferences 216, 03004 (2018). DOI: 10.1051/matecconf/201821603004 